\title{
UNIQUE FACTORIZATION MONOIDS AND DOMAINS
}

\author{
R. E. JOHNSON
}

Abstract. It is the purpose of this paper to construct unique factorization (uf) monoids and domains. The principal results are: (1) The free product of a well-ordered set of monoids is a uf-monoid iff every monoid in the set is a uf-monoid. (2) If $M$ is an ordered monoid and $F$ is a field, the ring $F[[M]]$ of all formal power series with well-ordered support is a uf-domain iff $M$ is naturally ordered (i.e., whenever $b<a$ and $a M \cap b M \neq \varnothing$, then $a M \subset b M$ ).

By a monoid, we shall always mean a cancellative semigroup with identity element. All rings are assumed to have unities, and integral domains and fields are not assumed to be commutative. If $R$ is an integral domain, then the multiplicative monoid of nonzero elements is denoted by $R^{\times}$. For any ring or monoid $A, U(A)$ will denote its group of units.

A monoid $M$ is called a unique factorization monoid (uf-monoid) iff for every $m \in M-U(M)$, any two factorizations of $m$ have a common refinement. That is, if $m=a_{1} a_{2} \cdots a_{r}=b_{1} b_{2} \cdots b_{s}$, then the $a$ 's and $b$ 's can be factored, say $a_{1}=c_{1} \cdots c_{i}, a_{2}=c_{i+1} \cdots c_{j}, \cdots, b_{1}$ $=d_{1} \cdots d_{k}, b_{2}=d_{k+1} \cdots d_{p}, \cdots$, in such a way that $c_{n}=d_{n}$ for all $n$. An integral domain $R$ is called a unique factorization domain (uf-domain) iff $R^{\times}$is a uf-monoid.

A monoid $M$ with relation < is said to be ordered by $<$ iff $<$ is a transitive linear ordering such that whenever $a<b$ then $a c<b c$ and $c a<c b$ for all $c \in M$. We call $M$ a positive monoid iff $M$ equals its positive cone $M^{+}=\{a \in M \mid a \geqq 1\}$. An ordered monoid $M$ is said to be naturally ordered (see [4, p. 154]) iff whenever $a M \cap b M \neq \varnothing$ and $b<a$, then $a M \subset b M$.

It is the purpose of this paper to show ways of constructing ufmonoids and uf-domains. The two principal results are as follows.

THEOREM 1. The free product of a well-ordered set of monoids is a uf-monoid iff every monoid in the set is a uf-monoid.

THEOREM 2. Let $M$ be an ordered monoid and $F$ be a field. The ring $F[[M]]$ of all formal power series with well-ordered support is a ufdomain iff $M$ is naturally ordered.

Received by the editors April 27, 1970.

AMS 1970 subject classifications. Primary 16A02, 20M25; Secondary 06A50.

Key words and phrases. Free products of monoids, unique factorization monoids, unique factorization domains, noncommutative power series rings. 
1. Unique factorization monoids. Another characterization of a uf-monoid is given by the following proposition.

Proposition 1. A monoid $M$ is a uf-monoid iff for all $a, b \in M$ such that $a M \cap b M \neq \varnothing$, either $a M \subset b M$ or $b M \subset a M$.

Proof. If $M$ is a uf-monoid and $a M \cap b M \neq \varnothing$, then $a a^{\prime}=b b^{\prime}$ for some $a^{\prime}, b^{\prime} \in M$ and $a=c_{1} \cdots c_{i}, a^{\prime}=c_{i+1} \cdots c_{n}, b=c_{1} \cdots c_{j}$, $b^{\prime}=c_{j+1} \cdots c_{n}$ for some $c_{i} \in M$. Hence, $a M \subset b M$ if $i \geqq j$ and $b M \subset a M$ if $i \leqq j$.

To prove the converse, let $m \in M$ have factorizations $m=a_{1} a_{2} \cdots a_{r}$ $=b_{1} b_{2} \cdots b_{s}$. Then $a_{1} M \cap b_{1} M \neq \varnothing$ and $a_{1}=b_{1} b_{1}^{\prime}$ (or $b_{1}=a_{1} a_{1}^{\prime}$ ) for some $b_{1}^{\prime} \in M$. Hence, $m_{1}=b_{1}^{\prime} a_{2} \cdots a_{r}=b_{2} \cdots b_{s}$. The desired conclusion now follows by induction on $r+s$.

In view of the result above, every naturally ordered monoid is a uf-monoid.

If an element $m$ of a uf-monoid $M$ can be factored into primes, say $m=p_{1} p_{2} \cdots p_{n}$, then the only other possible way to factor $m$ into primes is

$$
m=\left(p_{1} u_{1}\right)\left(u_{1}^{-1} p_{2} u_{2}\right) \cdots\left(u_{n-1}^{-1} p_{n}\right)
$$

where $u_{1}, u_{2}, \cdots, u_{n-1}$ are units. This follows readily from Proposition 1.

P. M. Cohn calls an integral domain $R$ with unity rigid [2] iff every nonzero, nonunit element of $R$ has unique factorization into primes. As he shows, a rigid domain $R$ is local (i.e., $R-U(R)$ is an ideal). In general, we have:

Proposition 2. If $R$ is a uf-domain, then $R$ is local.

Proof. If $a, b \in R^{\times}$and $a-b$ is a unit, then $a R+b R=R$ and hence $a R \cap b R \neq 0$. Therefore, $a R \subset b R$ or $b R \subset a R$ and either $a$ or $b$ is a unit. Hence, $R$ is a local ring.

There are many examples of uf-monoids. Trivially, a group or a free monoid is a uf-monoid. A valuation ring is a uf-domain according to Proposition 1. If $F[x, y]$ is the polynomial ring in noncommuting indeterminates $x$ and $y$ over a field $F$, then the set $H$ of homogeneous polynomials in $F[x, y]$ is a uf-monoid.

Given a well-ordered set $\left\{M_{i} \mid i \in \Gamma\right\}$ of monoids, we can form their direct product $\prod_{\Gamma} M_{i}$, their restricted direct product $\prod_{\Gamma}^{\prime} M$ $=\left\{a \in \prod_{\Gamma} M_{i} \mid \operatorname{card}\{i \in \Gamma \mid a(i) \neq 1\}<\infty\right\}$, and their free product $\prod_{\Gamma}^{*} M_{i}$.

Each $a \in \prod_{r}^{*} M_{i}, a \neq 1$, has a unique normal form 


$$
a=a_{i_{1}} a_{i_{2}} \cdots a_{i_{n}}, \quad a_{k} \in M_{k},
$$

where all $a_{i_{j}} \neq 1$ and $i_{j} \neq i_{j+1}$ for each $j$. Evidently $a$ is a unit iff each $a_{i_{j}}$ is a unit in $M_{i j}$. If $a$ is a nonunit, then $a$ can be expressed uniquely in the form $a=a^{\prime} u$ where $a^{\prime}=a_{i_{1}} \cdots a_{i_{r}}, u=a_{i_{r+1}} \cdots a_{i_{n}}, a_{i_{r}}$ is not a unit in $M_{i_{r}}$, and $u$ is a unit. We call $a^{\prime}$ right unit-free. If $r=n$, then $a^{\prime}=a$ and $u=1$. Similarly, $a=v a^{\prime \prime}$ for some unit $v$ and some left unit-free $a^{\prime \prime} \in \Pi_{\Gamma}^{*} M_{i}$. We are now ready to prove Theorem 1.

Proof of Theorem 1. If some $M_{i}$ in $\left\{M_{i} \mid i \in \Gamma\right\}$ is not a ufmonoid, then it is clear by Proposition 1 that $M=\prod_{r}^{*} M_{i}$ is not a uf-monoid. Next, assume that all $M_{i}$ are uf-monoids and that $a$, $b \in M$ with $a M \cap b M \neq \varnothing$. Clearly $a M \subset b M$ or $b M \subset a M$ if either $a$ or $b$ is a unit, so let us assume $a$ and $b$ are nonunits. Then $a=a^{\prime} u$ and $b=b^{\prime} v$ for some units $u, v$ and some right unit-free elements $a^{\prime}, b^{\prime}$. Let $a^{\prime}$ and $b^{\prime}$ have normal forms $a^{\prime}=a_{i_{1}} \cdots a_{i_{n}}$ and $b^{\prime}=b_{j_{1}} \cdots b_{j_{m}}$, and assume $m \leqq n$. Since $a^{\prime}$ and $b^{\prime}$ are right unit-free and $a^{\prime} M \cap b^{\prime} M$ $\neq \varnothing$, necessarily $i_{k}=j_{k}$ for $k \leqq m$ and $a_{i_{k}}=b_{i_{k}}$ for $k<m$. If $m<n$, $a_{i_{m}} \in b_{j_{m}} M_{j_{m}}$ and hence $a^{\prime} M \subset b^{\prime} M$. If $m=n$, then $a_{i_{n}} M_{i_{n}} \cap b_{j_{m}} M_{j_{m}} \neq \varnothing$ and either $a_{i_{n}} M_{i_{n}} \subset b_{j_{m}} M_{j_{m}}$ or $b_{j_{m}} M_{j_{m}} \subset a_{i_{n}} M_{i_{n}}$. Therefore, always $a^{\prime} M \subset b^{\prime} M$ or $b^{\prime} M \subset a^{\prime} M$. Since $a M=a^{\prime} M$ and $b M=b^{\prime} M$, we have proved that $M$ is a uf-monoid.

Proposition 3. Let $M$ be an ordered uf-monoid. Then $M$ is naturally ordered iff $M-M^{+} \subset U(M)$.

Proof. If $M$ is naturally ordered and $a<1$, then $1 M \subset a M$ and $a$ is a unit. If $M-M^{+} \subset U(M)$ and $a, b \in M$ with $a M \cap b M \neq \varnothing$ and $b<a$, then either $b M \subset a M$ or $a M \subset b M$. If $b M \subset a M$, then $b=a c$ for some $c<1$ and $b M=a M$ since $c$ is a unit. In either case, $a M \subset b M$. Hence, $M$ is naturally ordered.

A consequence of this proposition is that a positive uf-monoid is naturally ordered.

If $\left\{M_{i} \mid i \in \Gamma\right\}$ is a well-ordered set of ordered monoids, then their orders can be extended to $M^{\prime}=\prod_{\Gamma}^{\prime} M_{i}$, possibly in many different ways. One way is called the $\Gamma$-ordering: $a<b$ iff there exists $k \in \Gamma$ such that $a(i)=b(i)$ for all $i<k$ and $a(k)<b(k)$.

If $\left(M,<_{1}\right)$ and $\left(N,<_{2}\right)$ are ordered monoids and $M \stackrel{\theta}{\rightarrow} N$ is a homomorphism, then an ordering $<_{3}$ can be defined in $M$ by: $a<_{3} b$ iff either $\theta(a)<_{2} \theta(b)$ or $\theta(a)=\theta(b)$ and $a<_{1} b$. Clearly $\left(M,<_{3}\right)$ is an ordered monoid. For example, let $M^{\prime}=\prod_{\Gamma}^{\prime} M_{i}$ where each $M_{i}=\left\{x_{i}^{m} \mid m \in I\right\}$ and $I$ is $Z$ or $Z^{+}$. Then $M^{\prime} \stackrel{\text { deg }}{\rightarrow} Z$ is a homomorphism (deg = degree) and $M^{\prime}$ has the $(\Gamma, \mathrm{deg}$ )-ordering defined by: $a<b$ iff $\operatorname{deg} a<\operatorname{deg} b$ or $\operatorname{deg} a=\operatorname{deg} b$ and $a<b$ in the $\Gamma$-ordering. 
If $\left\{M_{i} \mid i \in \Gamma\right\}$ is a well-ordered set of ordered monoids, then the orderings in the $M_{i}$ can also be extended to an ordering in $\Pi_{\mathrm{r}}^{*} M_{i}$ [5]. In fact, the ordering in $\prod_{\Gamma}^{*} M_{i}$ can be chosen as an extension of any ordering in $\prod_{\Gamma}^{\prime} M_{i}$ in the sense that the natural epimorphism $\prod_{\Gamma}^{*} M_{i} \rightarrow \prod_{\Gamma}^{\prime} M_{i}$ will be order-preserving. We shall assume, henceforth, that whatever ordering we choose in $\Pi_{\Gamma}^{*} M_{i}$, it preserves the ordering in every $M_{i}$. Hence, if all the $M_{i}$ are positive monoids, then $\Pi_{r}^{*} M_{i}$ is also positive. It follows from Theorem 1 and Proposition 3 that a free product of positive uf-monoids is naturally ordered, no matter which order we choose in $\prod_{\Gamma}^{*} M_{i}$.

An example to show that the free product might be naturally ordered even if some of the monoids are not positive is as follows: Let $M_{1}=\left\{x^{n} \mid n \in Z^{+}\right\}, M_{2}$ be an ordered group, and $M=M_{1} * M_{2}$. If $M$ is ordered by an extension of this $(1,2)$-ordering in $M_{1} \times M_{2}$, then each $a \in M, a<1$, is in $M_{2}$ and hence is a unit. Thus, $M$ is naturally ordered by Theorem 1 and Proposition 3 . If the $(2,1)$ ordering is used in $M_{1} \times M_{2}$ and $a \in M_{2}, a<1$, then $a x<1$. Since $a x$ is not a unit, $M$ is not naturally ordered according to Proposition 3, although both $M_{1}$ and $M_{2}$ are naturally ordered.

For each monoid $M$, let $W_{0}(M)$ denote the set of all finite subsets of $M$; and if $M$ is ordered, let $W(M)$ denote the set of all well-ordered subsets of $M$. If $M$ is ordered and $A \subset M$, let inf $A$ denote the least element of $A$, if it exists. As usual, for any $A \subset M$ and $B \subset M$, we let $A B=\{a b \mid a \in A, b \in B\}$. The following result is proved in [3, Lemma 1].

Proposition 4. Let $M$ be an ordered monoid. For all $B, C \in W(M)$ and $d \in M, B \cup C$ and $B C$ are in $W(M)$ and $\{b \in B \mid d=b c$ for some $c \in C\}$ is in $W_{0}(M)$.

The following result is proved for ordered groups in [4, p. 135]. Since the proof does not seem to carry over readily to monoids, we have supplied a new proof.

Proposition 5. Let $M$ be an ordered monoid, $B \in W(M)$, $\bar{B}=\cup_{n=1}^{\infty} B^{n}$, and $Z_{a}=\left\{k \in Z^{+} \mid a \in B^{k}\right\}$ for each $a \in M$. If inf $B>1$, then $\bar{B} \in W(M)$ and card $Z_{a}<\infty$ for every $a \in \bar{B}$.

Proof. Assume that $\bar{B} \notin W(M)$, so that $\bar{B}$ contains infinite descending chains, and let $S$ be the set of all such chains in $\bar{B}$. Also, let $A=\left\{a \in B \mid a^{n}>C\right.$ for some $C \in S$ and some integer $\left.n>0\right\}$, where $a^{n}>C$ means $a^{n}>c$ for every $c \in C$. Finally, let $b$ be the least element of $A$ and $r$ be the least positive integer such that $b^{r}>C$ for some $C \in S$. 
If $b^{r}>C$, where $C=\left\{c_{k}\right\}$ and $c_{1}>c_{2}>\cdots$, then each $c_{k}$ $=b_{k 1} b_{k 2} \cdots b_{k n_{k}}$ for some $b_{k i} \in B$. Let $b_{k}=\max \left\{b_{k i} \mid i=1, \cdots, n_{k}\right\}$. Since $b_{k}^{n_{k}} \geqq c_{k}>c_{k+1}>\cdots$, evidently each $b_{k} \in A$. Thus, $b_{k} \geqq b$ for every $k$. We note that $r>1$; for if $b>c_{k}$ for each $k$, then $b>c_{k} \geqq b_{k}$ since $\inf B>1$. Now each $c_{k}$ has the form $c_{k}=p_{k} b_{k} q_{k}$ for some $p_{k}, q_{k} \in \bar{B}$ $\cup\{1\}$. Since $\left\{p_{k}\right\}\left\{b_{k}\right\}\left\{q_{k}\right\}$ is not well-ordered, either $\left\{p_{k}\right\}$ or $\left\{q_{k}\right\}$ is not well-ordered. Assume that $\left\{p_{k}\right\}$ is not well-ordered. Then $D \subset\left\{p_{k}\right\}$ for some $D \in S$. Since $b^{r}>c_{k}=p_{k} b_{k} q_{k} \geqq p_{k} b_{k} \geqq p_{k} b, b^{r-1}>p_{k}$ for every $k$. Thus, $b^{r-1}>D$ contrary to the minimality of $r$. Hence, $\bar{B} \in W(M)$.

Assume that card $Z_{a}=\infty$ for some $a \in \bar{B}$, and let $a$ be the least such element of $\bar{B}$. By assumption, $a=b_{k 1} b_{k 2} \cdots b_{k_{k} n_{k}}$ for some integers $2 \leqq n_{1}<n_{2}<\cdots$ and some $b_{k i} \in B$. We can assume, without loss of generality, that $b_{11} \leqq b_{21} \leqq b_{31} \leqq \cdots$. If we let $a_{k}=b_{k 2} \cdots b_{k n_{k}}$, then $a>a_{1} \geqq a_{2} \geqq a_{3} \geqq \cdots$. Hence, there exists an integer $m$ such that $a_{m}=a_{m+1}=a_{m+2}=\cdots$. Then card $Z_{a_{m}}=\infty$ contrary to the choice of $a$. Therefore card $Z_{a}<\infty$ for every $a \in \bar{B}$.

2. Ordered-monoid rings. In this section, $F$ is taken to be a ring with unity. Given a monoid $M$, an expression of the form

$$
f=\sum_{a \in M} \alpha_{a} a, \quad \alpha_{a} \in F,
$$

is called a formal power series in $M$ over $F$. As usual, the support of $f$ is defined by supp $f=\left\{a \in M \mid \alpha_{a} \neq 0\right\}$. The set

$$
F[M]=\left\{f=\sum \alpha_{a} a \mid \operatorname{supp} f \in W_{0}(M)\right\}
$$

becomes a ring if we define: for $f=\sum \alpha_{a} a, g=\sum \beta_{a} a, f+g=$ $\sum\left(\alpha_{a}+\beta_{a}\right) a, f g=\sum \gamma_{a} a$, where $\gamma_{a}=\sum_{b, c ; b c-a} \alpha_{b} \beta_{c}$. Then $F[M]$ is the usual monoid ring.

When we further assume $M$ is ordered, then we can also form the power series ring

$$
F[[M]]=\left\{f=\sum \alpha_{a} a \mid \operatorname{supp} f \in W(M)\right\}
$$

with operations as defined above. That $F[[M]]$ is a ring follows from Proposition 4 and the fact that supp $(f+g) \subset$ supp $f \cup$ supp $g$ and supp $f g \subset$ (supp $f$ )(supp $g$ ). Clearly we can consider $F \subset F[M]$ $C F[[M]]$. If $F$ is an integral domain, then so are $F[M]$ and $F[[M]]$. For if we define $\inf f$ to be inf supp $f$ whenever it exists, then it is 
clear that for all nonzero $f, g \in F[[M]]$, inf $f g=(\inf f)(\inf g)$ and hence $f g \neq 0$.

If $f \in F[[M]]$ and $\inf f>1$, then we see as in $[4$, p. 135] that $g=\gamma_{1} f+\gamma_{2} f^{2}+\cdots$ is a well-defined element of $F[[M]]$ for any $\gamma_{i} \in F$. Thus, if $f^{n}=\sum \alpha_{n a} a$, we take $g=\sum \beta_{a} a$ where $\beta_{a}=\sum_{n=1}^{\infty} \gamma_{n} \alpha_{n a}$. Since supp $g \subset U_{\text {supp }} f^{n} \subset U(\operatorname{supp} f)^{n}, g \in F[[M]]$ by Proposition 5 .

Proposition 6. Let $M$ an ordered monoid, $f=\sum \alpha_{a} a$ be a nonzero element of $F[[M]]$, and $d=\inf f$. Then $f$ is a unit iff $d$ is a unit of $M$ and $\alpha_{d}$ is a unit of $F$.

Proof. If $f$ has an inverse $f^{-1}=\sum \beta_{a} a$ and $c=\inf f^{-1}$, then $d c$ $=c d=1$ and $\alpha_{d} \beta_{c}=\beta_{c} \alpha_{d}=1$. Hence, $d \in U(M)$ and $\alpha_{d} \in U(F)$. Conversely, if $d \in U(M)$ and $\alpha_{d} \in U(F)$ then we can express $f$ in the form $f=\alpha_{d} d(1-h)$ for some $h \in F[[M]]$ with inf $h>1$. Then $k=h+h^{2}$ $+h^{3}+\cdots \in F[[M]]$ and we verify that $(1-h)(1+k)=1$ in the usual way. Therefore, $f$ has $(1+k) \alpha_{d}^{-1} d^{-1}$ as a right inverse. A similar argument shows $f$ also has a left inverse.

If $F$ is a field and $M$ is an ordered group, then this proposition is the well-known result $[4$, p. 137$]$ that $F[[M]]$ is a field.

We are now in a position to prove Theorem 2 .

Proof of Theorem 2. Assume that $R=F[[M]]$ is a uf-domain and $a, b \in M$ with $a<b$ and $a M \cap b M \neq \varnothing$. Then $a R \cap b R \neq 0$ and hence $a=b c$ or $b=a c$ for some $c \in M$. If $a=b c$, then $c<1$. Since $R$ is local by Proposition 2 and $(-c+1)+c \in U(R)$, either $c$ or $-c+1$ is a unit. In either case, $c$ is a unit of $M$ by Proposition 6. Therefore $b=a c^{-1}$ and always $b M \subset a M$. Hence, $M$ is naturally ordered.

Now assume $M$ is naturally ordered and $f, g \in R$ with $f R \cap g R \neq 0$ and inf $f=c \leqq d=\inf g$. Then $f g^{\prime}=g f^{\prime} \neq 0$ for some $f^{\prime}, g^{\prime} \in R$, and inf $f^{\prime}=c^{\prime} \leqq d^{\prime}=\inf g^{\prime}$. If $c^{\prime}$ is a unit, then $f^{\prime}$ is a unit by Proposition 6 and $g R \subset f R$. If $c^{\prime}$ is not a unit, then let $A=\left\{a \in \operatorname{supp} f^{\prime} \mid M a \cap M c^{\prime} \neq \varnothing\right\}$ and $B=\left\{a \in\right.$ supp $\left.g^{\prime} \mid M a \cap M c^{\prime} \neq \varnothing\right\}$. We can express $f^{\prime}=f_{1}^{\prime}+f_{2}^{\prime}$ and $g^{\prime}=g_{1}^{\prime}+g_{2}^{\prime}$, where supp $f_{1}^{\prime}=A, \operatorname{supp} f_{2}^{\prime}=\left(\operatorname{supp} f^{\prime}\right)-A, \operatorname{supp} g_{1}^{\prime}$ $=B$, and supp $g_{2}^{\prime}=\left(\operatorname{supp} g^{\prime}\right)-B$. Since $c^{\prime} \leqq a$ for all $a \in A \cup B$ and $M$ is naturally ordered, we must have $R a \subset R c^{\prime}$ for all $a \in A \cup B$. Thus, $f_{1}^{\prime}=h c^{\prime}$ and $g_{1}^{\prime}=k c^{\prime}$ for some $h \in U(R), k \in R$. Now $f k c^{\prime}+f g_{2}^{\prime}$ $=g h c^{\prime}+g f_{2}^{\prime}$, with $R c^{\prime} \cap R a=0$ for all $a \in\left(\operatorname{supp} f g_{2}^{\prime}\right) \cup\left(\operatorname{supp} g f_{2}^{\prime}\right)$. Therefore, $f k c^{\prime}=g h c^{\prime}, f k h^{-1}=g$, and $g R \subset f R$. This proves the theorem.

If $M_{i}=\left\{x_{i}^{n} \mid n \in Z^{+}\right\}$and $M=\prod_{\Gamma}^{*} M_{i}$, the free monoid with generators $\left\{x_{i} \mid i \in \Gamma\right\}$, then $M$ is naturally ordered for any ordering in $M$. Hence, by Theorem 2 , the ring $R=F[[M]]$ is a uf-domain for any field $F$. Clearly every nonzero nonunit $f \in R$ can be factored in to 
primes, since inf $f$ has only a finite number of factors in $M$.

If we take the $(\Gamma, \mathrm{deg})$-ordering in $M^{\prime}=\prod_{\Gamma}^{\prime} M_{i}$ and extend it to $M$, then $R$ consists of all formal power series in the $x_{i}$ over $F$ having only a finite number of terms of each degree. If the $\Gamma$-ordering is used in $M^{\prime}$ and extended to $M$, then we get a different ring $R$ of formal power series. While every $f \in R$ has only a finite number of terms of each degree, not all power series of this form are in $R$. For example, if $\Gamma=Z^{+}$and $f=x_{1}+x_{2}^{2}+x_{3}^{3}+\cdots, g=x_{1}+x_{1}^{2}+x_{1}^{3}+\cdots$, then $g \in R$ but $f \notin R$.

Another possible ordering in $M^{\prime}$ is the (dual $\Gamma$, deg)-ordering $<_{d}: a<_{d} b$ iff $\operatorname{deg} a<\operatorname{deg} b$ or $\operatorname{deg} a=\operatorname{deg} b$ and $b<a$ in the $\Gamma$-ordering. Clearly $<_{d}$ is an extension of the ordering in each $M_{i}$, and hence $<_{d}$ can be extended to an ordering in $M$.

The monoid $\left(M^{\prime},<_{d}\right)$ is well-ordered. For if $S \subset M^{\prime}$, $m=\min _{a \in S}(\operatorname{deg} a)$, and $T=\{a \in S \mid \operatorname{deg} a=m\}$, then the least element of $T$, if it exists, is the least element of $S$. Thus, we need only show that if $\operatorname{deg} S=m$ (i.e., $\operatorname{deg} a=m$ for all $a \in S$ ) then $S$ has a least element. If $m=0$ then $S=\{1\}$ and $S$ has a least element. Assume that every set $T$ of degree $m$ has a least element, and let $S$ be any set of degree $m+1$. If $i$ is the least element of $\Gamma$ such that $a(i) \neq 1$ for some $a=\prod_{\Gamma} a(i) \in S$ and $T=\{a \in S \mid a(i) \neq 1\}$, then the least element of $T$, if it exists, is the least element of $S$. Let $U=\left\{x_{i}^{-1} a \mid a \in T\right\}$, so that $U \subset M^{\prime}$ and $\operatorname{deg} U=m$. Now $U$ has a least element $b$ by the induction assumption. Clearly $x_{i} b$ is the least element of $S$. We conclude that $\left(M^{\prime},<_{d}\right)$ is well-ordered. Then $M$, with any ordering which is an extension of $<_{d}$, is also well-ordered. If $R=F[[M]]$, then $R$ is the set of all power series $\sum \alpha_{a} a$. Thus, $R$ is the power series domain considered by Cohn in [1].

The three different orderings in $M=\prod_{\Gamma}^{*} M_{i}$ considered above lead to three different power series rings $R=F[[M]]$. Each of these rings is a uf-domain for any field $F$ by Theorem 2. In particular, if the ordering $<_{d}$ is used we have another proof of Cohn's result in [1] that $R$ is rigid.

The example given above, $M=M_{1} * M_{2}$, where $M_{1}=\left\{x^{n} \mid n \in Z^{+}\right\}$, $M_{2}$ is any ordered group, and the $(1,2)$-ordering is taken in $M$ $=M_{1} \times M_{2}$, is naturally ordered. Hence, $R=F[[M]]$ is a uf-domain. Evidently $R$ is also rigid. If the $(2,1)$-ordering is taken in $M$, the resulting ring $P=F[[M]]$ is not a uf-domain since $M$ is not naturally ordered. Actually, $P$ is not even local. For if $a \in M_{2}, a<1$, and $b=-a x+1, c=a x$, then $b+c$ is a unit in $P$ although neither $b$ nor $c$ is a unit since inf $b=\inf c=a x$ is not a unit. 


\section{BIBLIOGRAPHY}

1. P. M. Cohn, Factorization in non-commutative power series rings, Proc. Cambridge Philos. Soc. 58 (1962), 452-464. MR 25 \#2092.

2. - - Hereditary local rings, Nagoya Math. J. 27 (1966), 223-230. MR 33 \#5663.

3. P. Conrad, Generalized semigroup rings, J. Indian Math. Soc. 21 (1957), 73-95. MR $20 \# 887$.

4. L. Fuchs, Partially ordered algebraic systems, Pergamon Press, New York; Addison-Wesley, Reading, Mass., 1963. MR 30 \#2090.

5. R. E. Johnson, Free products of ordered semigroups, Proc. Amer. Math. Soc. 19(1968), 697-700. MR 37 \#2864.

University of New Hampshire, Durham, New Hampshire 03824 